\title{
Small-Size Broadband Multi-Element Antenna for RF/Wireless Systems
}

\author{
B. A. Cetiner, Member, IEEE, L. Jofre, Member, IEEE, J. Y. Qian, Student Member, IEEE, and \\ F. De Flaviis, Member, IEEE
}

\begin{abstract}
We present a small-size broadband antenna specifically designed for integration with RF/wireless communication systems. The antenna is built on a printed circuit board and fed by a coplanar waveguide (CPW) to further allow easier integration with active and passive components of the communication system. The compact architecture of the antenna is suitable for multi-element scenarios for diversity implementations. Theoretical and experimental results showing impedance and radiation characteristics for both single- and multi-element antenna are given and discussed.
\end{abstract}

Index Terms-Diversity, small-size antenna, wireless system.

\section{INTRODUCTION}

$\mathbf{N}$ EW communication environments typically consists of a broad range of application classes of devices providing data, video, and audio services. The proliferation of cheaper, smaller, more compact, and higher performance mobile terminals has been fueling the effort to establish unified multiservice wireless local area networks (WLANS). Following this trend, antenna elements have to be compatible for integration with $\mathrm{RF} /$ wireless mobile terminals while at the same time they must satisfy desired impedance behavior and radiation characteristics for the intended application [1]. In particular, antennas with small size and architectures compatible with multi-element implementations are becoming key aspect of various communication systems that employ diversity techniques to improve the quality (bit error rate) or data rate of the communication. A perfect example to this scenario is a multi-input multi-output (MIMO) system, which is equipped with multi-element antennas at both transmitting and receiving ends. Some recent works have shown that MIMO systems in environments with rich scattering can provide dramatic increase in the wireless channel capacity by increasing the number of antennas [2].

Motivated by the features of next generation wireless communication systems, as stated above, we have aimed at developing a broadband small-size antenna architecture that can be integrated into an RF chip module, Personal Computer Memory Card International Association (PCMCIA) card in a laptop computer, or a peripheral component interconnect (PCI) card for a desktop. Furthermore, the architecture of the antenna is designed such

Manuscript received August 7, 2003; revised October 6, 2003. This work was supported in part by the U.S. Air Force, Edwards AFB, CA, under Grant F04611-03-C-0004.

B. A. Cetiner, J. Y. Qian, and F. De Flaviis are with the Electrical Engineering and Computer Science Department, University of California at Irvine, Irvine, CA 92697-2625 USA (e-mail: bedri@uci.edu).

L. Jofre is with the Department of Signal Theory and Communications, Technical University of Catalonia, Barcelona 08034, Spain.

Digital Object Identifier 10.1109/LAWP.2003.822211

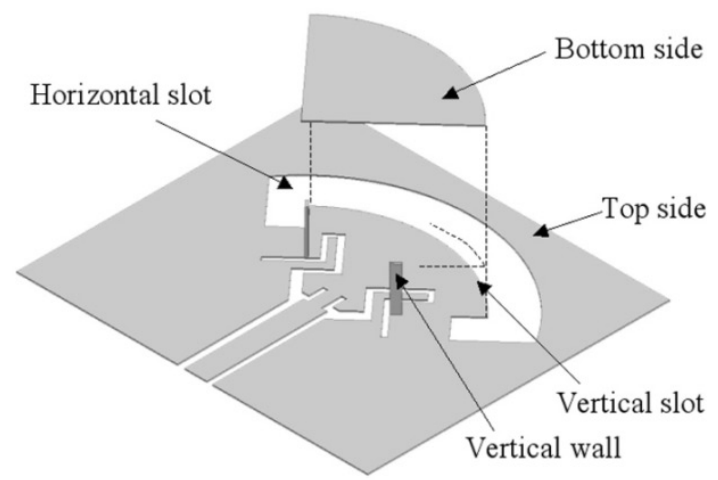

(a)

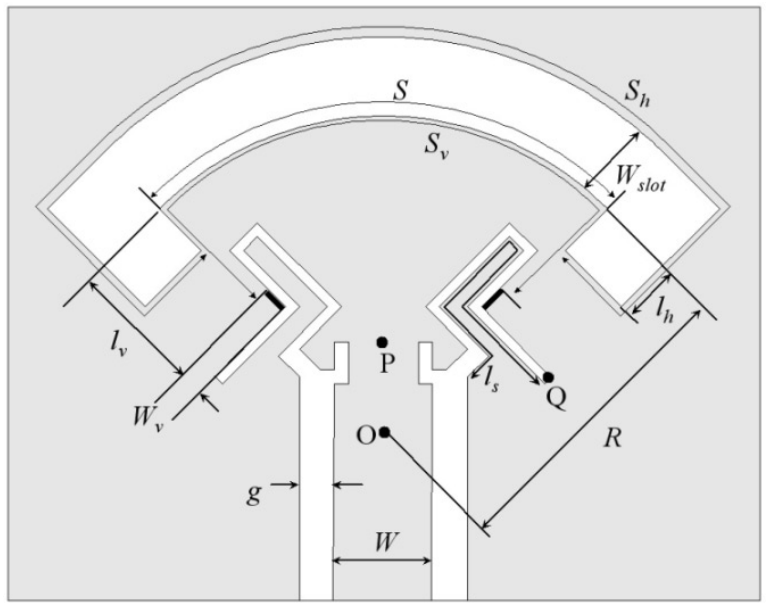

(b)

Fig. 1. Geometry of the proposed antenna. (a) Three-dimensional schematic (upside down). (b) Front view of the top side.

that it can be used in multi-element scenarios to exploit the benefits of diversity techniques [3].

\section{Structure AND Characterization OF THE ANTENNA}

\section{A. Geometry and Parametric Analysis}

Broadband miniature antennas, so called "dime" and "qdime" antennas, have recently been reported [4]. The qdime antenna with its quadric shape was obtained by modifying the geometry of the circularly shaped dime antenna in order to achieve an antenna architecture that efficiently uses the available real estate in multi-element scenarios. Both of these antennas are fed by coaxial cable and have three metallization levels among which two vertically stacked radial slots are formed. The antenna architecture proposed in this work has evolved from dime 


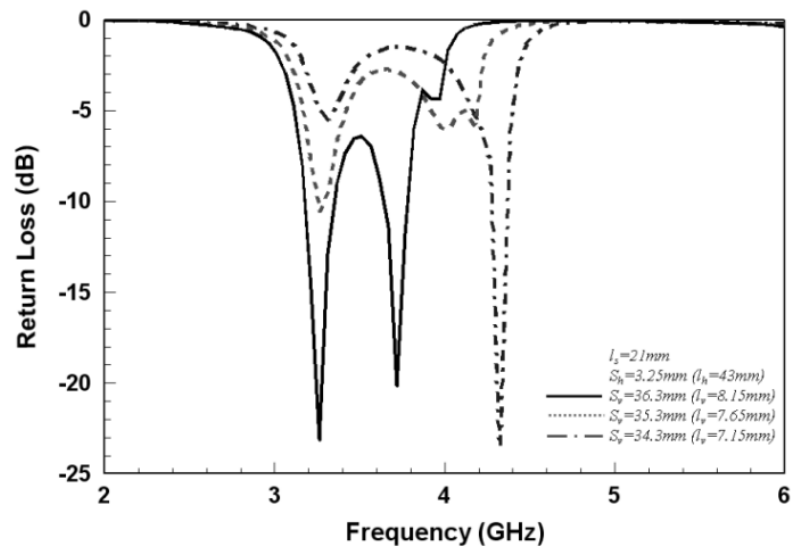

(a)

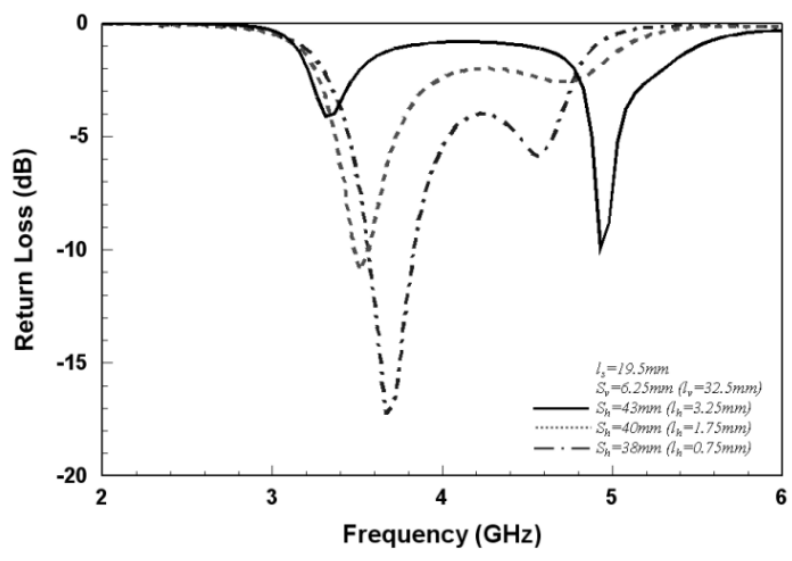

(b)

Fig. 2. Return loss of the antenna as a function of (a) vertical slot length $S_{v}$ and (b) horizontal slot length, $S_{h}$. $\left(W_{\text {slot }}=2.75 \mathrm{~mm}, S=20 \mathrm{~mm}\right.$ ).

and qdime antennas. The schematic of the antenna is depicted in Fig. 1(a) (for the clarity of the illustration the lower patch is shown as separated from the other parts of the structure). The antenna uses two-metallization level of a printed circuit board (PCB) and consists of a horizontal and a vertical slot as opposed to two vertical slots, which requires three-metallization level. This feature does not only make the antenna structure more compact but also allows easier fabrication on PCBs. In this new antenna geometry, the coaxial cable feed is also replaced by a CPW feed that offers number of advantages over other feed structures such as planar construction and suitability for integration with active or passive elements of the RF board. Fig. 1(b) shows the top side of the antenna, which contains the horizontal slot and feed circuitry, with each design parameter being designated a label in order to guide the design procedure summarized below. We define the equivalent length of the horizontal slot as $S_{h}+S+6 W_{\text {slot }}+2 l_{h}$. Two symmetrical slits with $l_{s}$ being defined as slit length are also etched into this plane. These slits force the feeding current to travel a longer path so as to reduce the size of the antenna area to a quarter of a circle of $\lambda / 5$ radius. For a good input impedance match, $l_{s}$ is chosen appropriately so that the feeding current travels a $\lambda / 4$ path [from $P$ to $Q$, see Fig. 1(b)] at the center design frequency. The bottom side of the antenna includes the quarter patch, which is connected to the top side through two symmetric vertical walls [see Fig. 1(a)]. The
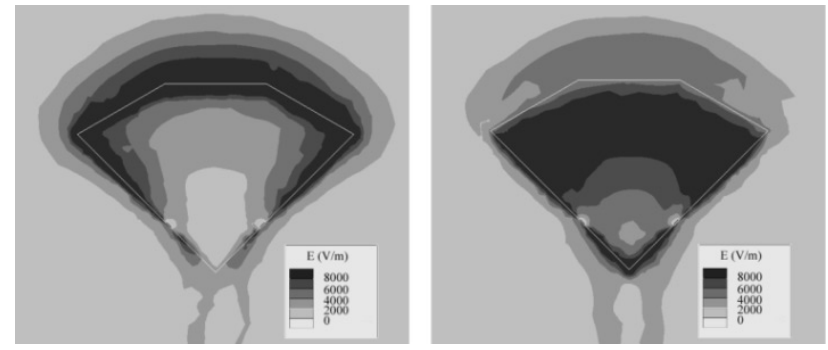

(a)
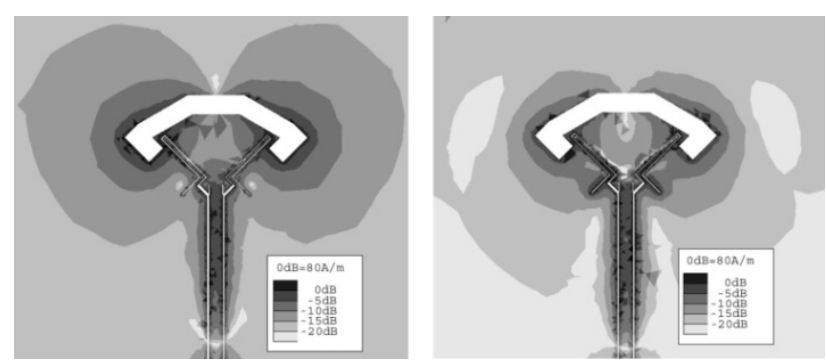

(b)

Fig. 3. Simulated distributions of current and electrical field on the antenna. (a) Electrical field distribution on the bottom surface of the antenna at the lower and upper resonant frequencies $f_{l}$ and $f_{u}$. (b) Current distribution on the top layer of the antenna at the lower and upper resonant frequencies, $f_{l}$ and $f_{u}$ $\left(f_{l}=3.4 \mathrm{GHz}\right.$ and $\left.f_{u}=3.8 \mathrm{GHz}\right)$.

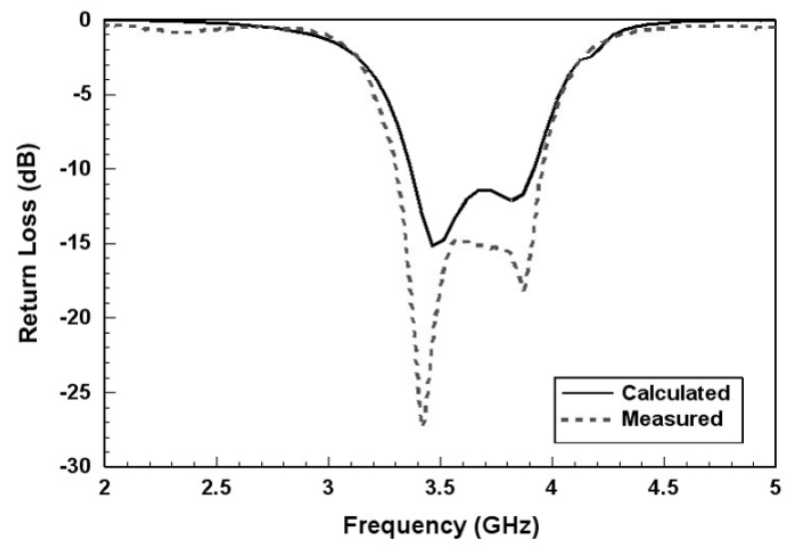

Fig. 4. Results for measured and calculated return loss of the fabricated prototype antenna. $R=12.75, S=20, l_{v}=8.1, l_{h}=2.1, S_{h}=38$, $S_{v}=36 ., W_{\text {slot }}=2.75, l_{s}=21$ (dimensions in millimeters).

vertical slot is formed between the two sides of the antenna with the thickness of the substrate $(\mathrm{h}=1.52 \mathrm{~mm})$. The length of this slot $S_{v}$ is determined by the two vertical walls $W_{v}$ and given by $S_{v}=S+2 l_{v}$. The CPW feed network is on the top side and has a width of $w$ and gap spacing of $g$ between the signal line and the ground plane, forming $50 \Omega$ characteristic impedance. To improve the matching level, the CPW feed line protrudes a short distance into the patch by two insets.

The structure of the antenna produces two different approximate $\lambda_{\text {eff }} / 2$ resonant frequencies, $f_{l}$ and $f_{u}$ determined by the lengths of the two slots $S_{h}$ and $S_{v}$, respectively

$$
S_{h, v} \cong \frac{c}{2 \sqrt{\varepsilon_{\text {reff }}} f_{l, u}}
$$

where $c$ is the speed of light in free space and $\varepsilon_{\text {reff }}$ represents the effective dielectric constant in the dielectric material. By prop- 


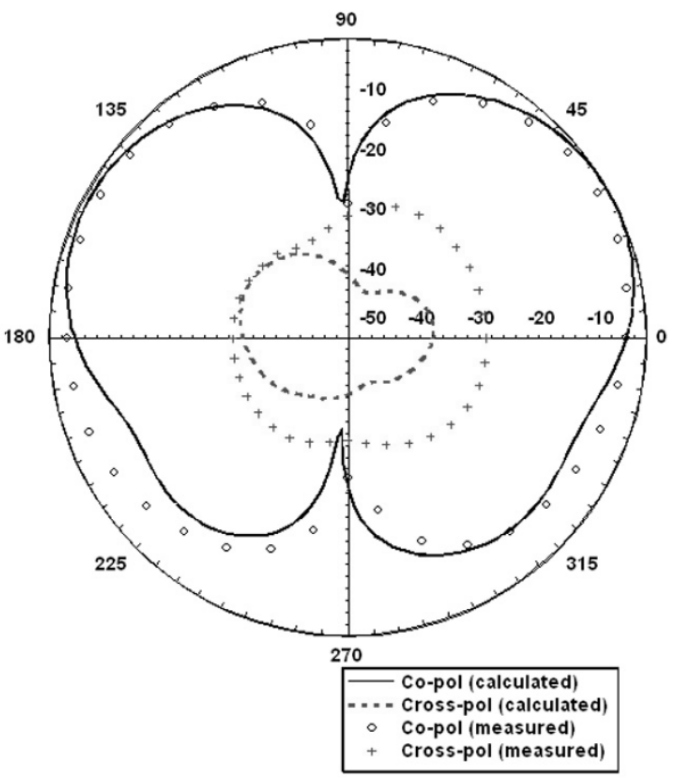

(a)

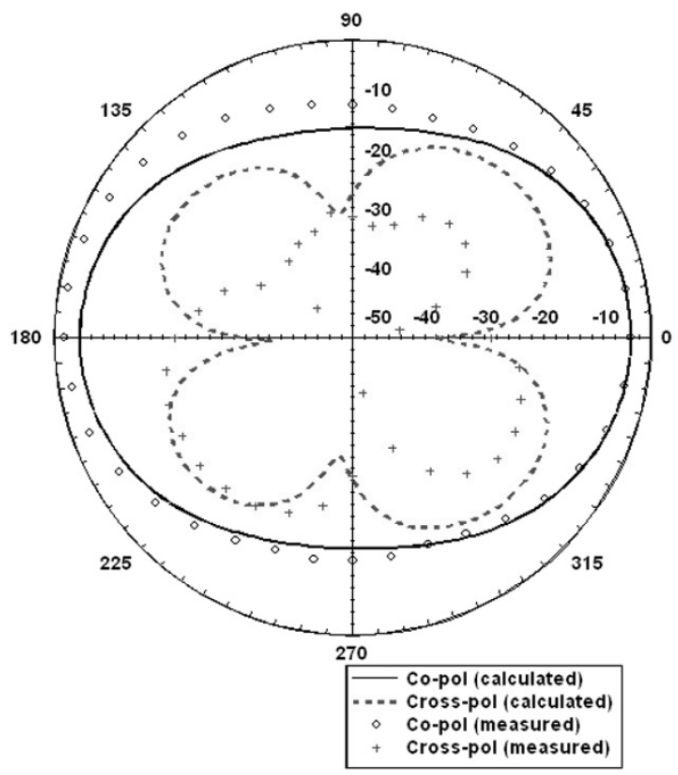

(b)

Fig. 5. Measured and calculated radiation patterns for the fabricated prototype antenna (a) $E$-plane. (b) $H$-plane.

erly locating these two frequencies, dual-band or single broadband behavior is obtained.

Ansoft HFSS8.5 is utilized to determine the influence of various antenna components. The return loss of the antenna as a function of frequency for different lengths of the vertical slot, $S_{v}$, and horizontal slot, $S_{h}$, are plotted in Fig. 2(a) and (b), respectively. The curves show that $S_{v}$ basically controls the upper resonant frequency, while $S_{h}$ influences both the lower and upper frequencies. The current and electrical field distributions were also studied to better understand the behavior and the interaction mechanisms between the different physical parts of the antenna. At the upper resonant frequency, as seen from Fig. 3(a), the electric field is confined into the cavity formed between top and bottom sides of the antenna. Due to this cavity

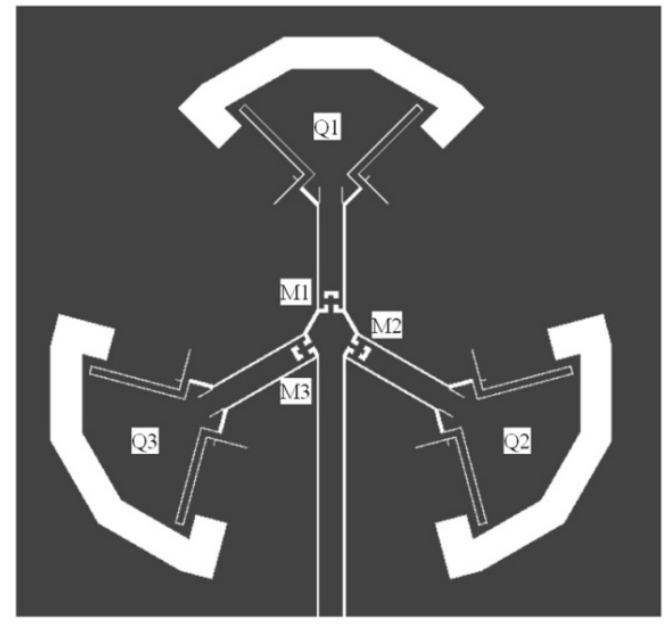

Fig. 6. Schematic of the first layer of the three-element antenna configuration. Antenna elements: $Q_{1}, Q_{2}, Q_{3}$; MEM actuators: $M_{1}, M_{2}, M_{3}$.

mechanism effect the upper resonant frequency is completely controlled by the length of the vertical slot as shown in Fig. 2(a). At the lower resonant frequency, although the current distribution around the horizontal slot, as seen in Fig. 3(b), increases significantly and determines the lower resonant frequency, it also plays a role on the upper resonant frequency, as seen in Fig. 2(b), due to the coupling mechanism with the vertical slot.

\section{B. Design, Simulation, and Measurement of a Prototype Antenna}

To validate the study and results obtained in the previous section we designed, simulated, and measured a S-band prototype antenna operating at the frequency band of 3-4 GHz. By following the design guidelines summarized above and according to (1), we determined the dimensions of the design variables: $S=20 \mathrm{~mm}, W_{\text {slot }}=2.75 \mathrm{~mm}$, $l_{h}=2.1 \mathrm{~mm}\left(S_{h}=40.7 \mathrm{~mm}\right), l_{v}=8.1 \mathrm{~mm}\left(S_{v}=36 \mathrm{~mm}\right)$, $l_{s}=21 \mathrm{~mm}(\mathrm{PQ}=20 \mathrm{~mm})$ so that two resonant frequencies $f_{l}=3.4 \mathrm{GHz}$ and $f_{u}=3.8 \mathrm{GHz}$ were located close enough resulting in single broadband behavior. The prototype antenna was fabricated on RT/Duroid 5695 microwave laminate with the thickness of $1.52 \mathrm{~mm}\left(\varepsilon_{\mathrm{r}}=2.55\right.$, loss tangent $\left.=0.0002\right)$ and fed through a subminiature version A (SMA) connector. Fig. 4 shows the measured and calculated return loss of the prototype antenna. The measured and simulated results agree well and comply with the broadband objective of the design with $25 \%$ impedance bandwidth despite its small size, $0.2 \lambda \times 0.2 \lambda$, compared to the conventional $0.5 \lambda \times 0.5 \lambda$.

Fig. 5 shows the radiation patterns of the antenna for $3.4 \mathrm{GHz}$ in the principal cuts, i.e., $E$ - and $H$-planes with good agreement between calculated and measured data except in the regions where the SMA connector plays a shadowing effect. This effect is especially visible for the cross-polar patterns where the radiation levels are lower. The behavior of the radiation pattern of this antenna with two semispherical sectors make it very appropriate for applications where conical coverage can be rotated around its perpendicular axis ( $z$-axis) to produce polarization diversity schemes. Radiation patterns for different frequencies within the input impedance bandwidth $(3.3-3.9 \mathrm{GHz})$ were also 


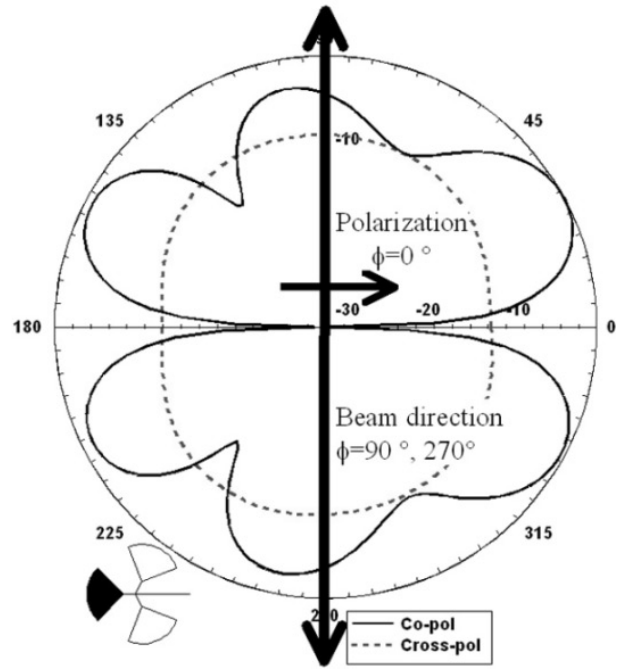

(a)

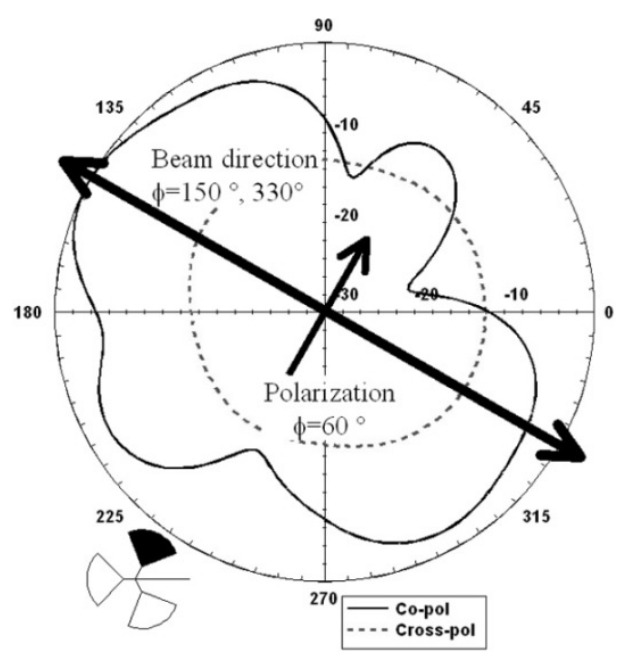

(b)

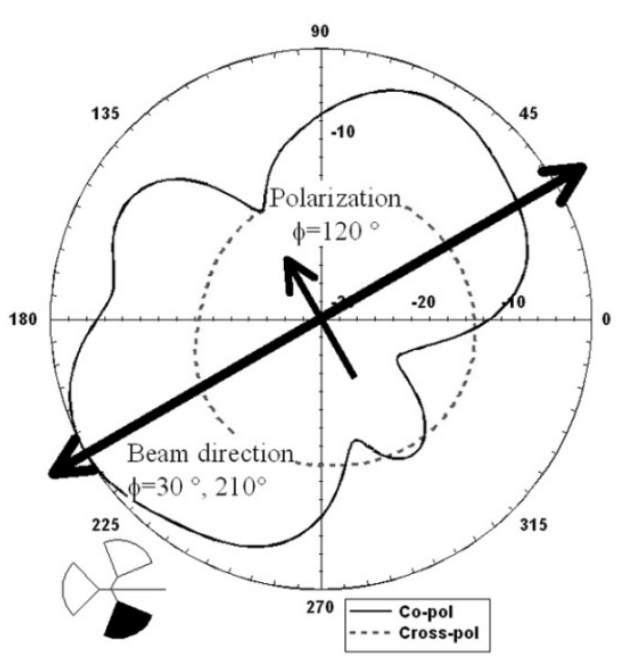

(c)

Fig. 7. Simulated co-pol radiation patterns from each antenna element in the $x-y$ plane when the MEM actuators are sequentially activated. (a) $M_{1}$ :on, $M_{2}$ :off, $M_{3}$ : off. (b) $M_{1}$ :off, $M_{2}$ :on, $M_{3}$ : off. (c) $M_{1}$ :off, $M_{2}$ :off, $M_{3}$ : on.

measured showing a similar behavior. The measured maximum gain at $3.5 \mathrm{GHz}$ was found to be $3.2 \mathrm{dBi}$.

\section{THREE-ELEMENT ANTENNA}

The proposed small-size antenna with its compact architecture, its low mutual coupling levels [4], and CPW feed circuitry is very well suited for multi-element antenna scenarios and offers the advantage of being integrated with other active and passive circuit components to achieve reconfigurability and multifunctionality. RF microelectromechanical system (MEMS) technology compatible with microwave laminate PCB substrates, recently developed within the author's group, can be employed to monolithically integrate MEMS with antenna systems to achieve desired multifunctionality [5]. Here we present an initial design employing three-element antenna for spatial-polarization diversity implementations. Shown in Fig. 6 is the schematic of the top view of the three-element antenna. The individual antennas are located around a hexagon and can be sequentially addressed using MEM actuators. This mechanism in turn provides different reception characteristics due to differences in polarization and spatial location of the individual antenna element and, thus, signals received over each antenna are decorrelated at some level. Decorrelated received signals then enable efficient diversity combining improving the quality of the communication. Fig. 7 shows the radiation patterns from each antenna element in the $x-y$ plane when switches connecting each antenna to the input port are sequentially activated. The three patterns represent three sets of diversity parameters on beam orientation $\left(\phi: 30^{\circ}, 90^{\circ}\right.$, $150^{\circ}$ ), polarization (linear: $0^{\circ}, 60^{\circ}, 120^{\circ}$ ), and spatial position (distance: $0.4 \lambda$ ), that can be simultaneously used to optimize the propagation capacity of the channel.

\section{CONCLUSION}

We aimed at developing a small-size broadband antenna fed by CPW compatible with RF/wireless system integration. The antenna was designed, fabricated, and characterized. Experimental impedance and radiation characteristics agree well with theoretical results. Design for three-element antenna suitable with MEMS integration was also introduced as an initial attempt to obtain multifunctional/reconfigurable antennas demanded by next generation communication systems.

\section{REFERENCES}

[1] IEEE Trans. Antennas Propagat-Special Issue Wireless Inform. Technol. Networks, vol. 50, May 2002.

[2] G. J. Foschini and M. J. Gans, "On limits of wireless communications in a fading environment when using multiple antennas," Wireless Personal Commun., vol. 6, no. 3, pp. 311-335, Mar. 1998.

[3] C. B. Dietrich, K. Dietze, J. R. Nealy, and W. L. Stutzman, "Spatial, polarization, and pattern diversity for wireless handheld terminals," IEEE Trans. Antennas Propagat., vol. 49, pp. 1271-1281, Sept. 2001.

[4] L. Jofre, B. A. Cetiner, and F. De Flaviis, "Miniature multi-element antenna for wireless communications," IEEE Trans. Antennas Propagat._Special Issue, vol. 50, pp. 658-669, May 2002.

[5] B. A. Cetiner, J. Y. Qian, H. P. Chang, M. Bachman, G. P. Li, and F. De Flaviis, "Monolithic integration of RF MEMS switches with a diversity antenna on PCB substrate," IEEE Trans. Microwave Theory Tech., vol. 51, pp. 332-335, Jan. 2003. 